J. Life Earth Sci., Vol. 8: 55-61, 2013

ISSN 1990-4827

http://banglajol.info.index.php/JLES

(C) 2013, JLES, RU

\title{
PERCEPTION LEVEL OF POTATO GROWERS TOWARDS PRODUCTION TECHNOLOGIES IN NORTHWEST BANGLADESH
}

\author{
A.B.M. Sharif Uddin ${ }^{1}$, M. Mostafizur Rahman ${ }^{2 *}$, M. Hasanul Kabir Kamaly ${ }^{3}$ and M. Bashirul Alam ${ }^{4}$ \\ ${ }^{1}$ Zila Parishad, Natore, Bangladesh; ${ }^{2}$ Department of Agronomy and Agricultural Extension, University of Rajshahi, \\ Rajshahi-6205, Bangladesh; ${ }^{3}$ Upazilla Agriculture Office, Tanore, Rajshahi, Bangladesh; ${ }^{4}$ Bangladesh Railway \\ (West), Rajshahi, Bangladesh. \\ Corresponding author: e-mail: mostafizur2001@yahoo.com
}

\begin{abstract}
The main focus of the study was to assess the extent of perception level of potato growers towards the potato production technologies. The study was carried out in the Rajshahi district which is considered as a part of the northwest area of Bangladesh. Two hundred thirty-two farmers were randomly selected as potato growers for data collection from twenty villages under the Upazilla namely, Mohonpur, Bagmara and Durgapur of Rajshahi district. A pre-tested interview schedule was used to collect the data from the respondents in July 2010 to February 2011. For measuring the perception of modern variety potato production technologies by the potato growers, 11 technologies for modern variety potato production were considered. Descriptive statistical measures such as number, percentage distribution, range, mean, standard deviation and coefficient of variation were used in describing the variable. More or less similar percentage (37\%) of the growers had medium and high perception whereas 26.29 percent low perception. Analysis indicated that among the extent of perception of modern eleven technologies use of quality seed was at top highest ranking by the perception adoption index of 253.88, whereas plant spacing was bottom lowest. The perception variations in different technologies will not be improved unless intensive and effective steps are taken to increase grower's access to various information sources of agriculture which increase the knowledge and then perception.
\end{abstract}

Keywords: Perception, production, technologies, potato.

সারাংশঃ আলু উৎপাদন প্রযুক্তির প্রতি আলু চাবীদের ধারণা স্তরের মাত্রা নিরুপণ করা এই অধ্যয়নের মূল উদ্দেশ্য। অধ্যয়নটি বাংলাদেশের উত্তর-পশ্চিমাংশ হিসেবে বিবেচ্য রাজশাহী জেলায় করা হয়েছিল। এই জেলার অন্তর্গত উপজেলা মোহনপুর, বাগমারা এবং দূর্গাপুর এর ২০টি গ্রাম থেকে দৈবায়িতভাবে নির্বাচিত ২৩২ জন আলু চাষীদের কাছ থেকে উপাত্ত সংগ্রহ করা হয়েছিল। জুলাই/২০১০-থেকে ফেব্রুয়ারি/২০১১ পর্যন্ত উত্তরদাতাদের কাছ থেকে পূর্বপরীক্ষিত সাক্ষাৎকার সূচী ব্যবহার করে উপাত্ত সংগ্রহ করা হয়েছিল। আলু চাষী কর্তৃক উন্নত জাতের আলু উৎপাদন প্রযুক্তির প্রতি ধারণা পরিমাপের জন্য ১১টি প্রযুক্তি বিরেচনা করা হয়েছিল। চলকের বর্ণনার জন্য বর্ণনামূলক পরিসংখ্যানিক পরিমাপক যেমন, সংখ্যা, শতকরা, শ্রেণীবদ্ধ, গড়, ট্ট্যান্ডার্ড ডেভিয়েশন এবং কোইফিসিয়েন্ট অব ভেরিয়েশন ব্যবহার করা হয়েছিল। ফলাফল থেকে দেখা যায় যে, আলু চাষীদের মধ্যম এবং উচ্চ ধারণার ক্যাটাগরী কম বেশি শতকরা (৩৭\%) একই রকম ছিল যেখানে শতকরা ২৬.২৯ ভাগ প্রযুক্তির প্রতি কম ধারণার আলু চাষী ছিল। ধারণার ইনডেক্স থেকে থেকে দেখা যায় যে, আলু উৎপাদনের ১১টি প্রযুক্তির মট্যে ‘গুণগত বীজ ব্যবহার’ এর ধারণার অবস্থান প্রথম ছিল এবং ‘গাছ লাগানোর দূরুত্ব’ ছিল ধারণার সর্বশেষ অবস্থান। আলু উৎপাদনের ১১টি প্রযুক্তির প্রতি ধারণার-পার্থক্য ততক্ষণ পর্যন্ত বৃদ্ধি পাবে, যতক্ষণ পর্যন্ত নিবিড় ও কার্যকরি পদক্ষেপ গ্রহণ করে আলু চাষীদের কৃষির তথ্যের বিভিন্ন উৎসের মট্যে প্রবেশ করানো না যায় যা তাদের জ্ঞান পরবর্তীতত ধারণার বৃদ্ধি ঘটায়।

\section{Introduction}

Agriculture is the economic backbone of Bangladesh as approximately 48.4 percent of its 145.9 million people directly and indirectly depends on it for subsistence and agriculture contributes about 20.60 percent of gross domestic product (BES, 2009). Potato (Solanum tubersosum) is the most important world's leading vegetable crop by virtue of its inherent potential for tonnage production, remunerative income and good nutritional value. It is a starchy, tuberous crop from the perennial Solanum tuberosum of the Solanace family also known as the nightshades. Among the agricultural crops, potato (Solanum tubersosum) has become an important crop for both farmers and consumers in Bangladesh. Now a days, it is the third largest food crop in Bangladesh and production are increasing day by day. Bangladesh has made a visible progress in potato production during 1950-51 to 2007-08. Area under potato has increased to double and production has also risen to double during the same period due to favorable soil and climate (Rashid, 1987). The average yields in northern region of Bangladesh which is considered to be one of the most important production areas for potato production, is estimated to be around 15-18 tons per ha. Potato is ideally suited to places where land is limited and labour is abundant, conditions that characterize much of the developing world. Moreover, the potato is a highly productive crop. It produces more food per unit area and per unit time than wheat, rice and maize. Factors that influence potato yield and quality include cultivar, soil type, weather conditions, water management, fertilization, plant population, seed size, pests and diseases. The potato gives a high return compared to other crops in Bangladesh and it is cultivated during the Rabi season from November to March.

Perception is the process concerned with the acquisition and interpretation of information from one's environment (Maddox, 1995). Perception refers to the process of creating meaningful patterns from raw sensory information. Hence, our perceptual processes enable us to understand and make sense of the sensations that we are continually experiencing; otherwise, even the most mundane task would become impossible. However, experiencing meaningful patterns 
in the jumble of sensory information is what we mean by perception (Morris, 1993). Perception is an activity through which an individual becomes aware of objects around him and events taking place. Perception of the same situation may differ from individual to individual due to differences in their experiences and cognitive domain. The expectations, needs and ways of thinking influence how and an individual interprets what he observes. Most of the farmers of Bangladesh are illiterate, they have perceptual problems towards the adoption of any technology. Farmer's production performance does not only depend on physical and technology resources available to them but also his perception condition. The proper management of potato cultivation is fully dependent upon the ability of the farmer's knowledge, skill, attitude which influences the perception. Perception can help farmers to adopt potato production technologies efficiently and to get higher production. Considering the above facts the present study was taken in hand to measure the perception level of potato growers towards the potato production technologies.

\section{Materials and Methods}

The study was carried out in the Rajshahi district which is considered as the part of northwest Bangladesh. Three Upazilla namely, Mohonpur, Bagmara and Durgapur from this district also selected purposively due to consider their production and area under potato cultivation. Finally, six villages from Mohonpur, ten from Bagmara and four from Durgapur were selected randomly as a locale of the study. Total number of farmers who has at least 0.13 hectare of land for potato cultivation in these twenty villages was 1547 which constituted population of the study, 232 farmer as potato growers were selected randomly as the respondents covering 15 percent of active population. Data were collected during the month of July 2010 to February 2011. Necessary secondary data were also collected from different sources in addition to primary data. Descriptive statistical measures such as number, percentage distribution, range, mean, standard deviation and coefficient variation were used in describing the variable.

Measurement of perception of modern variety potato production technologies: Perception of an individual regarding any technology plays a very important role in the innovation decision process. Grower's perception on potato production technologies was the main variable in this study. The production technologies of potato production consists of 11 items which are, (i) well land preparation, (ii) cultivation of modern variety, (iii) fertilizer dose, (iv) fertilizer application method, (v) irrigation, (vi) plant protection measures, (vii) quality seed, (viii) inter cultural operations, (ix) planting time, (x) seed size and (xi) planting space. This investigation attempted to ascertain the extent of perception towards 11 technologies of modern variety potato production by using a 4 point modified Likert type scale like strongly agree, agree, disagree, and strongly disagree and the corresponding scores were 3 , 2, 1, and 0,respectively (Kamaly, 2011). Perception for determining with 11 technologies was actually measured through selected attributes like i) relative advantages, ii) compatibility, iii) demonstration, iv) complexity, v) high investment and vi) risk involvement of the potato growers regarding potato production technologies. Finally, perception of an individual was measured through computation of scores of those six attributes of each technology. Among the 11 technologies, well land preparation which could from 0 to 18, where 0 indicated no perception and 18 indicated high perception of well land preparation. The same way was followed for other 10 technologies.

Overall perception score of all technologies: Thus the overall scores of perception of potato production technologies could range from 0-198 (Table 1.), where 0 indicated no perception and 198 assumed for highest range for perception. But this possible range of perception was definitely found above 0 and less than 198 which was real calculated range for measuring actual elasticity of perception. And there by, the elasticity was categorized as low, medium and high according to their range of score distribution as found as minimum to maximum level.

Comparative perception of eleven selected production technologies of potato production practices: For comparing the extent of perception of all selected technologies as a factor of perception determinants it was required to compute a perception index for each of the eleven technologies. The perception index for a certain technology was computed by using the following formula:

Perception index $=\mathrm{Pn} \times 0+\mathrm{Pl} \times 1+\mathrm{Pm} \times 2+\mathrm{Ph} \times 3$ $\mathrm{Pn}=$ Percentage of potato growers having no perception.

$\mathrm{Pl}=$ Percentage of potato growers having low perception.

$\mathrm{Pm}=$ Percentage of potato growers having medium perception. $\mathrm{Ph}=$ Percentage of potato growers high perception.

Perception index of a technology could be range from 0 to 300 , where 0 indicates no perception and 300 for maximum perception. 
Table 1 Scoring system of perception of potato production technologies

\begin{tabular}{|l|l|l|c|}
\hline \multicolumn{1}{|c|}{ Item of production technologies (11) } & \multicolumn{1}{|c|}{ Measuring scale } & \multicolumn{1}{c|}{ Attributes (6) } & $\begin{array}{c}\text { Possible } \\
\text { scores range }\end{array}$ \\
\hline 1) Well land preparation & & & $0-18$ \\
2) Cultivation of modern variety & Strongly Agree=3 & Relative advantage & $0-18$ \\
3) Fertilizer dose & Compatibility & $0-18$ \\
4) Fertilizer application method & Agree=2 & Demonstration & $0-18$ \\
5) Irrigation & Complexity & $0-18$ \\
6) Plant protection measures & Strongly disagree=0 & High investment & $0-18$ \\
7) Use of quality seed & (4 point Likert type scale) & & $0-18$ \\
8) Intercultural operations & & & $0-18$ \\
9) Planting time & & & $0-18$ \\
10) Seed size & & & $0-18$ \\
11) Planting space & & $0-18$ \\
\hline \multicolumn{2}{|r|}{} & & $0-198$ \\
\hline
\end{tabular}

\section{Results and Discussion}

Perception of modern variety potato production technologies by the growers in the study area has been cited sequentially in this part and presented in Table 2.

Perception of well land preparation: Soil fertility and land productivity is followed by good ground preparation by well prepared land through successive plowings, with associated harrowing and mixing with manures and fertilizers. Eliminating all root-weeds is desirable during land preparation. Computed land preparation scores as a part of perception of potato production technologies by the respondents ranged from 05 to 16 against a possible score of 0 to 18 . The mean, standard deviation and co-efficient of variation of this calculated scores were 9.67, 3.11 and 9.68, respectively. Based on perception of well prepared land scores, the respondents were classified into three categories as sown in Table 2. Analysis indicated that 43.96 percent of the potato growers had low perception of well prepared land compared to 33.18 \& 22.84 percent had medium and high perception, respectively. The presented data also revealed that more than threefourth $(77.14 \%)$ of potato growers had medium to low perception of well land preparation. It might be due to constraints of labor force and unavailability of required production inputs like power tiller, manures, fertilizers etc. Correct potato husbandry can be an arduous task in some circumstances but a well prepared land along with a little grace from the weather is a good beginning of underground tuber formation and growth from planted quality seed of modern variety.

Perception of modern variety cultivation: Genetically improved line of potato (Solanum tuberosum) is the basis of modern variety which is the key factor to expect higher yields. The computed scores for perception of modern variety cultivation of the respondents ranged from 04 to 15 against a possible score of 1 to 15 . The mean, standard deviation and coefficient of variation of this calculated scores were 12.07, 2.87 and 8.27, respectively. Based on perception of modern variety cultivation scores, the respondent growers were classified into three categories as sown in Table 2. Data presented in Table 2 indicates that 60.34 percent of the potato growers had high perception of modern variety cultivation compared to 28.45 percent medium and 11.21 percent having low perception. This finding has similarity with the findings of Muttaleb (1995). Area coverage and continuation of cultivation of any high yielding modern variety is a very important issue to maximize a sustainable production. More than sixty percent having high perception shows a clear indication of importance of modern variety cultivation given by the potato growers. It might be due to relative advantage of potato as short duration crop with higher yield and economic gain. So, it is drawing an attention of extension authorities for their devotion in the scope of expansion of modern variety cultivation.

Perceptions of balanced fertilizer doze: Computed balanced fertilizer doze scores ranged from 05 to 16 against a possible score of 0 to 18 . The mean, standard deviation and co-efficient of variation of this calculated scores were 10.28, 2.88 and 8.32, respectively. Based on perception of balanced fertilizer doze scores, the respondents were classified into three categories as sown in Table 2. Analysis indicated that majority had medium (50.43\%) perception compared to 24.57 percent high and 25.00 percent had low perception of balanced fertilizer dose technology as determined by selected attributes of perception. Observed scores also revealed that, three-fourths $(75.00 \%)$ of the potato growers belonged to medium to high category. It might be due awareness of growers towards plant nutrients as a vital factor of potato production. 
Perception of recommended fertilizer application method:

Computed scores of the respondents, however ranged from 5 to 16 against a possible score range of 0 to 18 with a mean value 10.89 , standard deviation 2.60 and co-efficient of variation 8.78. The potato growers were classified into three categories on the basis of perception of recommended fertilizer application method scores as shown in the Table 2. The presented data in Table 2 shows that 49.14 percent respondents had medium perception of recommended fertilizer application method compared to 18.96 percent having low perception and 31.90 percent having high perception. Data also revealed that more than four-fifths (81.04\%) respondents had medium to high perception. Therefore, special attention needs to be given to help the potato growers in the use of this technology of recommended fertilizer application method as perceived by the potato growers.

Perception of optimum irrigation: The possible range of optimum irrigation scores was 0 to 18 where the computed irrigation score was found 6 to 16 as perceived of potato production technologies by the respondent growers. The mean, standard deviation and co-efficient of variation of the calculated scores were 11.86, 2.54 and 6.46, respectively. Perception categories of potato growers are shown in Table 2 on the basis of optimum irrigation scores. Table 2 shows that 55.60 percent of the potato growers had high perception of optimum irrigation compared to 38.36 percent medium and 06.03 percent having low perception. The presented data also revealed that majority had high perception of optimum irrigation. Due to irrigation scheme of Barind Multipurpose Development Authority (BMDA) in the study area might be a mental make up of perceiveness of importance of irrigation to the crop field by the potato growers. Research has proved that irrigation can increase the yield of potato to a remarkable extent (Anonymous, 2000).

Table 2 Distribution of potato growers on the basis of perception scores

\begin{tabular}{|c|c|c|c|c|c|c|}
\hline \multirow{2}{*}{$\begin{array}{l}\text { Perception of } \\
\text { technologies }\end{array}$} & \multirow{2}{*}{ Perception categories (scores) } & \multicolumn{2}{|c|}{ Potato growers } & \multirow{2}{*}{ Mean } & \multirow{2}{*}{$\begin{array}{l}\text { Standard } \\
\text { deviation }\end{array}$} & \multirow{2}{*}{$\begin{array}{l}\text { Co-efficient of } \\
\text { variation }\end{array}$} \\
\hline & & Number & Percent & & & \\
\hline \multirow{3}{*}{$\begin{array}{l}\text { Well land } \\
\text { preparation }\end{array}$} & Low perception (05-08) & 102 & 43.96 & \multirow{3}{*}{9.67} & \multirow{3}{*}{3.11} & \multirow{3}{*}{9.68} \\
\hline & Medium perception (09-13) & 77 & 33.18 & & & \\
\hline & High perception (13-16) & 53 & 22.84 & & & \\
\hline \multirow{3}{*}{$\begin{array}{l}\text { Cultivation of } \\
\text { modern variety }\end{array}$} & Low perception(05-08) & 26 & 11.21 & \multirow{3}{*}{12.07} & \multirow{3}{*}{2.87} & \multirow{3}{*}{8.27} \\
\hline & Medium perception(09-13) & 66 & 28.45 & & & \\
\hline & High perception(13-16) & 140 & 60.34 & & & \\
\hline \multirow[t]{3}{*}{ Fertilizer dose } & Low perception (5-8) & 58 & 25.00 & \multirow{3}{*}{10.28} & \multirow{3}{*}{2.88} & \multirow{3}{*}{8.32} \\
\hline & Medium perception (9-12) & 117 & 50.43 & & & \\
\hline & High perception (13-16) & 57 & 24.57 & & & \\
\hline \multirow{3}{*}{$\begin{array}{l}\text { Fertilizer } \\
\text { application } \\
\text { method }\end{array}$} & Low perception(5-8) & 44 & 18.96 & \multirow{3}{*}{10.89} & \multirow{3}{*}{2.60} & \multirow{3}{*}{8.78} \\
\hline & Medium perception (9-12) & 114 & 49.14 & & & \\
\hline & High perception(13-16) & 74 & 31.90 & & & \\
\hline \multirow{3}{*}{$\begin{array}{l}\text { Optimum } \\
\text { irrigation }\end{array}$} & Low perception(6-8) & 14 & 06.03 & \multirow{3}{*}{11.86} & \multirow{3}{*}{2.54} & \multirow{3}{*}{6.46} \\
\hline & Medium perception (9-12) & 89 & 38.36 & & & \\
\hline & High perception(13-16) & 129 & 55.60 & & & \\
\hline \multirow{3}{*}{$\begin{array}{l}\text { Plant protection } \\
\text { measures }\end{array}$} & Low perception(6-8) & 39 & 16.81 & \multirow{3}{*}{10.70} & & \\
\hline & Medium perception (9-12) & 117 & 50.43 & & 2.72 & 7.40 \\
\hline & High perception(13-16) & 76 & 32.76 & & & \\
\hline Use of quality & Low perception (5-8) & 19 & 8.19 & & & \\
\hline seed & Medium perception (9-12) & 69 & 29.74 & 12.39 & 2.61 & 6.84 \\
\hline & High Perception (13-16) & 144 & 62.07 & & & \\
\hline Intercultural & Low perception (6-8) & 36 & 15.52 & & & \\
\hline operations & Medium perception (9-12) & 113 & 48.71 & 11.02 & 2.67 & 7.17 \\
\hline & High Perception(13-16) & 83 & 35.77 & & & \\
\hline Planting time & Low perception (6-8) & 61 & 26.29 & & & \\
\hline & Medium perception (9-12) & 62 & 26.73 & 11.24 & 2.95 & 8.74 \\
\hline & High Perception(13-16) & 109 & 46.98 & & & \\
\hline Seed size & Low perception (5-8) & 117 & 50.43 & & & \\
\hline & Medium perception (9-12) & 86 & 37.07 & 9.58 & 2.65 & 7.03 \\
\hline & High Perception(13-16) & 29 & 12.50 & & & \\
\hline Planting spacing & Low perception(6-8) & 124 & 53.45 & & & \\
\hline & Medium perception (912) & 82 & 35.34 & 9.02 & 2.45 & 6.00 \\
\hline & High Perception (13-15) & 26 & 11.21 & & & \\
\hline
\end{tabular}


Perception of plant protection measures: The possible range of perception on plant protection measures scores was 0 to 18 where the computed score was found 6 to 16 as perceived of potato production technologies by the respondent growers. The mean, standard deviation and co-efficient of variation of the calculated scores were $10.70,2.72$ and 7.40 , respectively. Perception categories of potato growers are shown in Table 2. Data presented in Table 5.6 indicates that the highest proportion (50.43\%) of the growers had medium perception compared to 16.81 percent had low and 32.76 percent had high perception of plant protection measures technology. The findings also show that highest proportion (67.24\%) of the respondents had medium to low perception.

Perception on use of quality seed: Perception scores of quality potato seed of the growers ranged from 5 to 16 , against the possible range of 0 to 18 . The average score was found to be 12.39 with the standard deviation of 2.61 and co-efficient of variation 6.84. Based on quality seed scores, the respondents were classified into three categories as shown in Table 2 which indicates that majority of the respondents had high perception (62.07\%) of quality potato seed compared to 8.19 percent low and 29.74 medium perception of quality potato seed. The data also reveals that near about onethird of the potato growers (37.93\%) had medium to low adoption. Seed is a basic indicator of expected yield per unit area. No other factor can ensure maximum production until quality seed is planted. Therefore, high perception in this regard demands availability of quality potato seed as a technology of milestone of expected production of potato.

Perception of intercultural operations: Intercultural operations scores of the potato grower respondents could range from 0 to 18 . Computed scores of the respondents, however ranged from 6 to 16 with an average value of 11.02, standard deviation and coefficient of variation were 2.67 and 7.17, respectively. The potato growers were classified into three categories on the basis of observed scores of perception of intercultural operations as shown in the Table 2. The presented data in the said table shows that 48.71 percent respondents had medium perception of intercultural operations as compared to 15.52 percent having low perception and 35.77 percent having high perception. Data also revealed that about two-thirds (64.23\%) respondents had medium to low perception. Therefore, special attention needs to be given to motivate the potato growers in increasing their perceiveness of intercultural operations as recommended technology. It is also appreciated that proper training program on intercultural operations of potato cultivation might be the positive response to aware of plant protection measures.

Perception of planting time: Scores of computed planting time as observed by the respondent potato growers ranged from 06 to 16 against the possible range of 0 to 18 , the mean being 11.24 with standard deviation and co-efficient of variation were 2.95 and 8.74, respectively. The potato growers were classified into three categories on the basis of observed scores of perception of planting time as shown in Table 2. It shows that 46.98 respondents had high perception of optimum planting time of potato as compared to 26.29 percent having low perception and 26.73 percent having medium perception. Data also revealed that majority of 53.02 percent respondents had medium to low perception. It might be due to the fact that timely supply of quality seed depends on mainly seed suppliers or traders according to their capability or capacity to import or multiplication of potato seed which is beyond control of potato growers even on seed suppliers. Land preparation might be affected by cultivated previous crop staying in the field and moisture percentage in the soil of potato field also linger planting time. Potato growers usually don't perceive any idea of monoculture of crop production. They usually tried their best to adjust maximum number of crop in a year by rotation even with relay crop, mixed crop or inter crop in the same season. So, planting time as a technology matter might have not perceived as suggested specially for potato cultivation.

Perception of recommended seed size: Computed scores of the respondents, however ranged from 5 to 16 with an average of 9.58 and standard deviation and coefficient of variation 2.65 and 7.03, respectively. The potato growers were classified into three categories on the basis of observed scores of perception of recommended seed size as shown in Table 2 which indicates that 50.43 percent respondents had low perception of recommended seed size as compared to 37.07 percent having medium perception and 12.50 percent having high perception. Apparently, it seems that a seed may be termed as a tuber which retains sprouts of seed vigor. Every grower wants to ensure a seedling from each planted potato seed. So, their prime concern is devoted to good tuber seed rather a piece of cut tuber with optimum number of sprouts. This mental orientation might be the reflection of scores given by the potato growers in the study area. 
Perception of potato plant spacing: Perception scores of plant spacing of the potato growers ranged from 6 to 15 against the possible range of zero $(0)$ to 18 . The mean was found to be 9.02 with the standard deviation of 2.45 and co-efficient of variation 6.00. Based on observed spacing scores, the respondents were classified into three categories as shown in Table 2 which indicates that majority of the respondents had low perception of plant spacing scores compared to 35.34 percent medium and 11.21 percent high perception of potato plant spacing scores. The data also reveals that near about nine-tenths $(88.79 \%)$ of the potato growers had medium to low perception. Optimum plant spacing is required to ensure proper growth. Findings of the study indicates that perception of potato plant spacing is remaining behind which would be necessary to improve through motivational training program.

Overall perception of potato growers towards modern variety potato production technologies: In order to have a clear understanding of the combined perception of the eleven selected potato production technologies, an overall perception score was computed for each of the respondent by adding the score of 11 modern potato production technologies. Computed overall perception scores of the growers ranged from 72 to 163 . The mean value was being 118.78 with standard deviation and coefficient of variation 24.96 and 623.36, respectively. Based on the observed scores, potato grower farmers were classified into three categories such as "low" perception, "medium" perception and "high" perception as shown in Table 3.

Table 3 Distribution of the potato growers according to their overall perception of modern potato technologies

\begin{tabular}{|c|c|c|c|c|c|}
\hline \multirow{2}{*}{ Categories overall perception (scores) } & \multicolumn{2}{|c|}{ Potato growers } & \multirow{2}{*}{ Mean } & \multirow{2}{*}{$\begin{array}{l}\text { Standard } \\
\text { deviation }\end{array}$} & \multirow{2}{*}{$\begin{array}{l}\text { Co-efficient of } \\
\text { variation }\end{array}$} \\
\hline & Number & Percent & & & \\
\hline Low perception (72-100) & 61 & 26.29 & \multirow{4}{*}{118.78} & \multirow{4}{*}{24.96} & \multirow{4}{*}{623.36} \\
\hline Medium perception (101-131) & 86 & 37.07 & & & \\
\hline High perception (132-163) & 85 & 36.64 & & & \\
\hline Total & 232 & 100 & & & \\
\hline
\end{tabular}

Data contained in Table 3 indicates that slight majority (37.07\%) had medium perception compared to high (36.64\%) and low (26.29 percent) perception. But almost three-fourths $(73.71 \%)$ of the potato growers had medium to high perception. The perception scores measured by selected attributes showed that potato growers perceived the technologies to a reasonable extent which was a positive indication of potato production. But the recent past record of potato growth did not match according to their perceiveness. If the actual orientation in use of innovations of potato technologies are found then the real scenario could be ascertained. Unless the status of adoption is determined it would not be clear the gap of perception and production trend by use of technologies.

Table 4 Rank order of eleven potato production technologies according to their perception index

\begin{tabular}{|l|l|c|c|}
\hline Sl. No. & Modern Technologies & Perception Index & Rank Order \\
\hline 1 & Well land preparation & 178.84 & 09 \\
\hline 2 & Cultivation of modern variety & 249.13 & 03 \\
\hline 3 & Fertilizer doze & 199.57 & 08 \\
\hline 4 & Fertilizer application method & 212.94 & 07 \\
\hline 5 & Optimum Irrigation & 249.55 & 02 \\
\hline 6 & Plant protection measures & 215.95 & 06 \\
\hline 7 & Use of quality seed & 253.88 & 01 \\
\hline 8 & Intercultural operations & 220.25 & 05 \\
\hline 9 & Planting time & 220.69 & 04 \\
\hline 10 & Seed size & 162.07 & 07 \\
\hline 11 & Planting spacing & 157.76 & 10 \\
\hline
\end{tabular}

Comparative perception of eleven selected production technologies of potato production practices: The computed indices of the eleven modern technologies ranged from 157.76 to 253.88. The eleven modern technologies have been arranged in rank order on the basis of their perception indices in Table 4 . Analysis of data in Table 4 indicates that among the extent of perception of modern eleven technologies use of quality seed was at top highest ranking by the perception adoption index of 253.88. The average yield increase from the use of good quality seed is 30 to 50 percent compared to farmers' seeds (Wang, 2008). Quality indicators of potato seed have two dimensions: the biological attributes (biological quality) and the 
appearance attributes (commercial quality). Biological quality is crucial for productivity, whereas commercial quality mainly affects seed price. The $2^{\text {nd }}$ position was optimum irrigation (249.55). Hassan et al. (2002) showed that yield of potato increases up to $25 \%$ with proper irrigation. The perception index of modern variety cultivation, planting time, intercultural operation, plant protection measures, fertilizer application method, fertilizer doze, well land preparation land, seed size and plant spacing were 249.13, 220.69, 220.25, 215.95, 212.94, 199.57, 178.84, 162.07 and 157.76, respectively as shown in Table 4. Ranked of planting spacing was the lowest. Due to small farm size farmers are not interested to follow optimum planting space. It is the common scenario in Bangladesh.

\section{Conclusion}

Overall perception of eleven technologies of modern variety potato production technologies was medium to low. Thus, the perception on of potato technologies was low among the potato growers in general. Higher perception is expected for better adoption as well as for maximum yield. Hence, for the achievement of experimented yield by potato experts or scientists, the level of potato grower's perception should also be high. At present yield of potato per hectare in Bangladesh is around 16 tons compared to world average 40 tons. Hence, there is a great potential for increasing the yield of potato provided farmers readiness to perceiveness and response to use of appropriate technologies i.e. adoption. The findings lead to the conclusion that overall perception by the potato growers of the study area will not be improved unless intensive and effective steps are taken to increase their access to various information sources of agriculture. Growers' perception was comparatively lower as rated by them in case of plant spacing, seed size and well land preparation; but their perception is higher in case of use of quality seeds, optimum irrigation and modern variety cultivation. Hence, it may be concluded that without improving knowledge by adequate training facilities and ensuring availability of those required inputs in time at farm level growers will not get ready to perceive those innovations.

\section{References}

Anonymous, 2000. Krishi Projucti Hatboi. Bangladesh Agricultural Research Institute, Joydevpur, Gazipur, Bangladesh, pp. 8-9.

BES. 2009. Bangladesh Economical Survey. Economical Advisory Section, Finance Division, Ministry of Finance, Government of the Peoples Republic of Bangladesh. June 2009.

Hassan AA, Sarkar AA, Ali MH \& Karim NN. 2002. Effect of Deficit Irrigation at Different Growth Stage on the Yield of Potato. Pakistan J. Biol. Sci., 5: 128-134

Kamaly MHK. 2011. Farmers’ Perception on the Harmful Effects of Using Agro-chemicals in Rice Production. Ph.D. Thesis, Institute of Bangladesh Studies, University of Rajshahi, Rajshahi. Bangladesh, pp. 91-96.

Maddox GL. 1995. The Encyclopedia of Agony (2nd edn.). New York: Springer Publishing Company, Inc.

Morris CG. 1993. Psychology-An introduction. New Jersey: Prentice Hall.

Muttaleb MA. 1995. Farmers' Preference Matching and Adoption of Modern Rice Cultivation Practices in Haor Area of Northeast Bangladesh. Ph.D.Thesis. Department of Agricultural Extension Education. Bangladesh Agricultural University, Mymensingh, Bangladesh, pp. 208-212.

Rashid MM. 1987. Potato Production in Bangladesh. A Consultancy Report Prepared for FAO Regional Office for Asia and the Pacific, Bangkok, Thailand. Tuber Crops Research Center, Bangladesh Agricultural Research Institute, Joydevpur, Gazipur, Bangladesh, pp. 1-11.

Wang F. 2008. The Importance of Quality Potato Seed in Increasing Potato Production in Asia and the Pacific Region. Workshop to Commemorate the International Year of Potato 2008. Bangkok, Thailand, 6 May 2008, pp 46-53.

Manuscript received on 28 November 2013 and revised on 19 December 2013 